\title{
An Efficient Simulation-Based Search Method for Reliability-Based Robust Design Optimization of Mechanical Components
}

\author{
M. MAYDA \\ Karamanoglu Mehmetbey University, 70100, Karaman, Turkey, E-mail: mmayda@kmu.edu.tr \\ cross $^{\text {ref }}$ http://dx.doi.org/10.5755/j01.mech.23.5.15745
}

\section{Introduction}

Nowadays optimization under uncertainty (OUO) in engineering design, which is also known to be stochastic design optimization, has gained an ever increasing importance. OUO can be grouped into three classes: reliability-based design optimization (RBDO), robust design optimization (RDO) and reliability-based robust design optimization (RBRDO). RBDO focuses on deducing a failure probability of a system consisting of random design variables or parameters with known probability distributions. Numerous research on RBDO has been conducted. For example, Yang et al. [1] proposes a methodology for RBDO to handle uncertainties in the offshore wind turbines design process. For that purpose, they constructed a finite element (FE) model and generated some design points from the model. Using a surrogate or an approximate model built based on the design points, they implemented the reliability analysis in a reasonable computational cost. Similarly, there are a lot of research in the literature [2-4]. Nevertheless, the variation in the objective function is often not minimized in RBDO; instead, it investigates the probabilities at the tails of the distribution function [5]. RDO concentrates to minimize the variability in a system without eliminating the source of the variability; thus to make the system insensitive to effects of the fluctuations $[6,7]$. The earliest approach on RDO is known to be Six Sigma Quality [8] and Taguchi method $[9,10]$. These methods aims to reduce the output variation in the input-output system and accordingly to improve the product quality. Most of recent researches on RDO have been concentrating on multi-objective optimization using surrogate models and various algorithms such genetic algorithm and Particle Swarm Optimization (PSO) algorithm [11-14]. However, Forouzandeh Shahraki and Noorossana [15] express that the merits of RDO and RBDO should be combined to ensure both reliability and robustness of the designs, which is the main aim of RBRDO method. Although the concept of RBRDO is not new, the RDRDO method is gaining an ever increasing importance when seen in the recent literature [15-17]. In this work, the RBRDO method is taken as a base optimization procedure.

Applying all of these optimization methods in design problems, by its nature, requires more computationalintensive efforts compared to the deterministic case [18]. To mitigate this issue, surrogate models built based on design inputs and outputs have been widely used in recent years [19]. The use of surrogate models instead of real models drastically reduces the computational cost of the design optimization methods [20]. Accordingly, the frequently used surrogate models are Kriging model, Artificial Neural Network (ANN) model and Polynomial models. However, there are still several challenges to be overcome. First, the existing formulations and implementations of RBRDO are complex to apply them to design problems [21]. Second, despite the significant improvements in the computational cost of building an approximate model, a computationally efficient method of optimum search is needed to enhance the RBRDO process. To address these issues, in this work, an efficient simulation-based search method for RBRDO problems is proposed. The proposal to the first issue is a simple RBRDO definition by implementing it through MonteCarlo Simulation (MCS), which is one of the simplest and the most powerful methods [19]. MCS grounds on randomly generating samples based on stochastic characteristics of random variables and evaluating the sample set generated against given criteria. Also, as a surrogate model, ANN is used to build an approximate model representing relationships between design variables and responses. Therefore, the combination of MCS and ANN is considered to make the optimization process more efficient and effective. The proposal to the second issue is that a search vector based on correlation coefficients between design variables and responses is considered with the aim of accurately selecting searching direction and step lengths towards the robust design point in a short time.

The reminder of this paper is organized as follows: In Section 2, the definition of design optimization under uncertainty is described. In Section 3, the proposed simulationbased search method is explained in detail. In Section 4, a numerical example is illustrated on the design of a car window handle to show the effectiveness and efficacy of this method. In Section 5, a conclusion and future work are stated.

\section{Definition of design optimization under uncertainty}

Design optimization can be classified as two categories: deterministic design optimization and stochastic design optimization. The definitions of two categories vary depending on where to use them. A deterministic design problem can be generally formulated in the literature as [18]:

$$
\left.\begin{array}{l}
\operatorname{Min} f(x) \\
\text { subject to }: h_{j}(x) \leq 0, \quad j=1, \ldots, n_{\text {ineq }}, \\
g_{k}(x)=0, k=1, \ldots, n_{e q}, \\
x^{L} \leq x \leq x^{U}
\end{array}\right\}
$$

where $f$ is stands for the objective function. $x=\left\{x_{1}, x_{2}, \ldots, x_{N d}\right\}$ denotes the vector of deterministic design variables. $N d$ is the number of deterministic design variables. $h$ and $g$ represent constraint functions. $n_{\text {ineq }}$ is the number of inequality constraints. $n_{e q}$ is the number of 
equality constraints. $x^{U}$ and $x^{L}$ are the upper and lower limits of the design variables respectively.

The stochastic design optimization deals with optimizing designs under uncertainty; therefore, this kind of optimization can be considered as reliability-based design optimization (RBDO), robust design optimization (RDO) and reliability-based robust design optimization (RBRDO). The definition of a generic RBDO process is given by

$$
\begin{aligned}
& \operatorname{Min} f\left(x, \mu_{\theta}\right) \\
& \text { subject to: } \\
& \left.\begin{array}{rl} 
& P\left(L_{m}(x, \theta)\right) \geq R_{m}, \quad m=1, \ldots, n_{l s} \\
\text { or } \quad & P\left(L_{m}(x, \theta)\right) \leq 1-R_{m}, \\
& x^{L} \leq x \leq x^{U},
\end{array}\right\}
\end{aligned}
$$

where $f$ stands for the objective function. $\theta=\left\{\theta_{1}, \theta_{2}, \ldots, \theta_{N r}\right\}$ is the vector of random design variables or parameters. $\mathrm{Nr}$ is the number of random design variables. $\mu_{\theta}$ represents the mean value of a random design variable. $L_{m}(\cdot)$ stands for limit-state functions indicating margins of failures of probability of a design. $n_{l s}$ is the number of limit-state functions. $R_{m}$ is the level of target reliability, which is a percentile value.

RDO focuses on the variation of the objective function. A generic definition of RDO is as follow [22]:

$$
\left.\begin{array}{l}
\text { Min }(\mu(f(x, \theta)), \sigma(f(x, \theta))) \\
\text { subject to : } \\
L_{m}(x, \theta), m=1, \ldots, n_{l s}, \\
x^{L} \leq x \leq x^{U},
\end{array}\right\}
$$

where $\mu(\cdot)$ and $\sigma(\cdot)$ are the mean and standard deviation values of the objective function, $f$, respectively.

Nevertheless, to account for both the reduction in variability of a system and mitigating failure probability stemming from uncertainties, the RBRDO process, based on RBDO and RDO, is typically followed by using the definition [23]:

$$
\begin{aligned}
& \operatorname{Min}(\mu(f(x, \theta)), \sigma(f(x, \theta))) \\
& \text { subject to: } \\
& \left.\begin{array}{rl} 
& P\left(L_{m}(x, \theta)\right) \geq R_{m}, m=1, \ldots, n_{l s}, \\
\text { or } \quad & P\left(L_{m}(x, \theta)\right) \leq 1-R_{m}, \\
& x^{L} \leq x \leq x^{U} .
\end{array}\right\}
\end{aligned}
$$

In this work, the RBRDO definition is considered as a base model.

\section{The proposed simulation-based search method}

In this section, the proposed simulation-based method is explained in five steps, given by a flowchart in Fig. 1.

1. Prior to starting the process, a design problem is chosen and analyzed. Herein, the main challenge is to deal with the experimental or simulated data instead of analytical data because there is no any analytical equation or relationship between the design inputs and outputs. Thus, in the scope of this work, the attention is given to the design data obtained from FEM (Finite Element Method) simulations. In this step, a sample set consisting of design variables $\left(D V_{i}, i=1, \ldots, n_{D V}\right)$ and design responses $\left(D R_{j}\right.$, $\left.j=1, \ldots, n_{D R}\right)$ are obtained from FEM simulations. $n_{D V}$ and $n_{D R}$ are the number of design variables and design responses, respectively.

\section{Initial design}

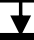

Generation of a sample set consisting of design variables $\left(D V_{i}\right)$ and design responses $\left(D R_{j}\right)$

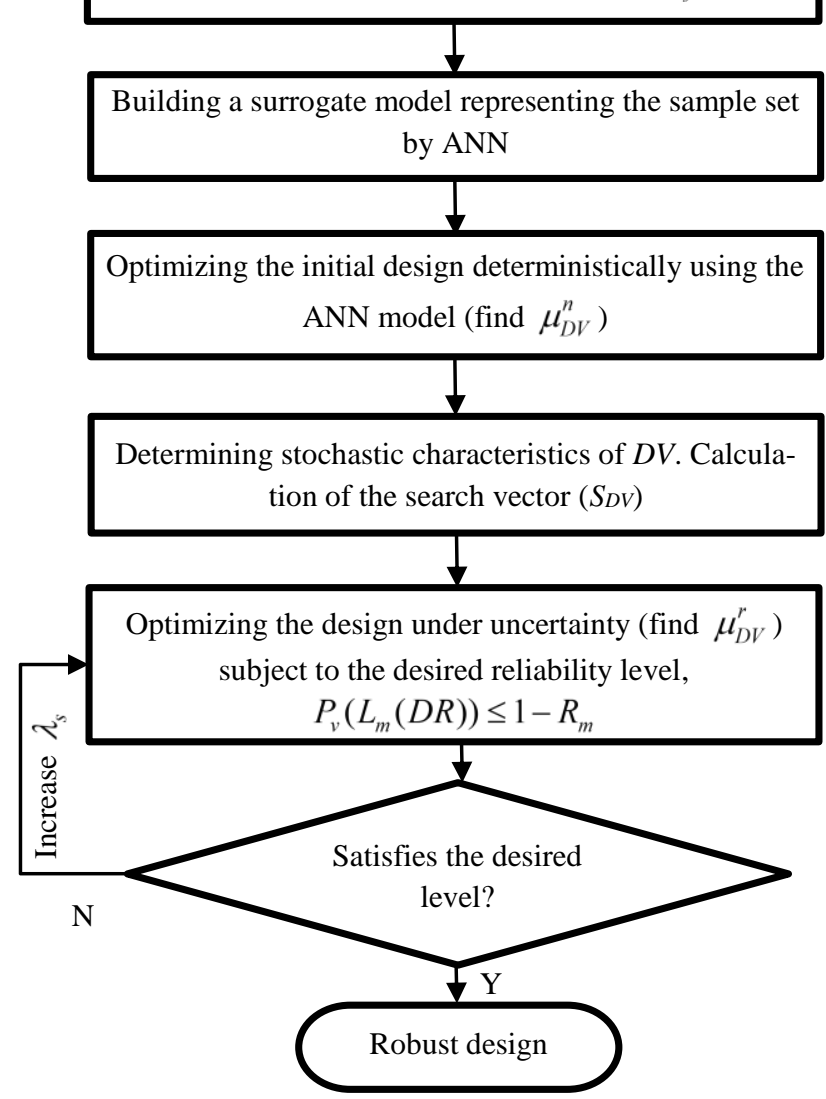

Fig. 1 The flowchart of the proposed simulation-based method

2. A surrogate model is needed to represent the linear or non-linear relationship between the $D V$ and $D R$ in most of RBRDO problems. The ANN model is considered as a surrogate model so as to model the non-linear relationship between the $D V$ and $D R$ obtained from FEM simulations. To validate this model, the sample set is divided into two groups; one group is used for training the ANN model and the other for testing the model in terms of $\mathrm{R} 2$ value.

3. To reduce the process time of uncertainty-based modelling and optimization, a deterministic optimization of the initial design within ranges with predetermined lower and upper bounds is realized by using the well-known sampling method, MCS. The optimization process yields a nominal or mean value of $i$ th design variable of the first optimal design to be found deterministically prior to robust design optimization, which is denoted as $\mu_{D V_{i}}^{n}$ and considered as a starting design point for the next steps.

4. In this step, the stochastic characteristics of the $D V$, such as distribution type, standard deviations of the $D V$, are specified depending on the existing knowledge or experience. The standard deviation values of $D V, \sigma_{D V}$ are assumed to be subjected to a known coefficient of variation ( 
$\delta_{D V}$ ), also so-called as COV. Therefore, $\sigma_{D V}$ are calculated by the formula: $\sigma_{D V_{i}}=\delta_{D V_{i}} \cdot \mu_{D V_{i}}^{n}$, which is the standard deviation of $i$ th design variable. Other task is to calculate a search vector $\left(S_{D V}\right)$ that has descent or ascent directions leading to optimal design points. The $S_{D V}$ can be found by

$$
\left.\begin{array}{l}
S_{D V_{i}}=\delta_{D V_{i}} \mu_{D V_{i}}^{n} \rho_{w_{D V_{i}}}, \\
\rho_{w_{D V_{i}}}=\sum_{j=1}^{n_{D R}} \rho_{i j} W p_{j}, \quad \sum_{j=1}^{n_{D R}} W p_{j}=1,
\end{array}\right\}
$$

where $S_{D V_{i}}$ is the coefficient of variation for $i$ th design variable. $\rho_{w_{D V_{i}}}$ is a relatively weighted correlation coefficient corresponding to each design variable. $\rho_{i j}$ is the correlation coefficient between $i$ th design variable and $j$ th design response. $W p_{j}$ is a weight value of relative importance (as percentage) for $j$ th design response, which can be determined by the decision maker. Also the summation of the $W p$ is assumed to be 1 .

5. If the RBRDO definition given in Eq. (4) is reformulated in line with the aim of this research, the new form of the definition is as follows:

$$
\left.\begin{array}{c}
\operatorname{Min}\left(\mu_{D V}^{r}, \sigma_{D V}, \lambda_{s}\right) \quad \lambda_{s}=1, \ldots, m, \\
\mu_{D V}^{r}=\mu_{D V}^{n}{ }^{T}+S_{D V}{ }^{T} \cdot \lambda_{s}, \\
\text { Subject to }: P_{v}\left(L_{m}(D R)\right) \leq 1-R_{m},
\end{array}\right\}
$$

here $\mu_{D V}^{r}$ is the vector of mean values of $D V$ of the robust design. $\lambda_{s}$ denotes a scaling factor that determines the minimum step length for the $S_{D V}$ for each evaluation of the limit-state or constraint function. $m$ is a maximum integer or decimal value that is needed to be found in the optimization loop because the $m$ might help to search for a minimum

$\mu_{D V}^{r} . P_{v}$ represents the probability of violation of the constraint or limit-state functions.

\section{Numerical example}

In this section, the design optimization of a car window handle under uncertainty is considered to show how to use the proposed method in practice, and accordingly to clearly demonstrate its effectiveness and efficacy. The initial geometrical dimensions of the handle are indicated in Fig. 2. Herein, the letters $V$ and $F$ in dimensions refer a design variable and fixed dimension, respectively. Totally, five design variables (two distances (two equal lengths, $6 \mathrm{~mm}$ ), two radiuses (R97 and R100) and thickness $(4.4 \mathrm{~mm}))$ are selected for the design optimization. With regard to boundary conditions, there is an applied load on the right side of the handle, its $\mathrm{x}$ and $\mathrm{y}$ components are $F_{x}$ of 150 $\mathrm{N}$ and $F_{y}$ of $400 \mathrm{~N}$, and a fixed support on the other side of the handle (Fig. 3). The safety factor and mass of the handle under the boundary conditions are accepted as design responses $(D R)$.

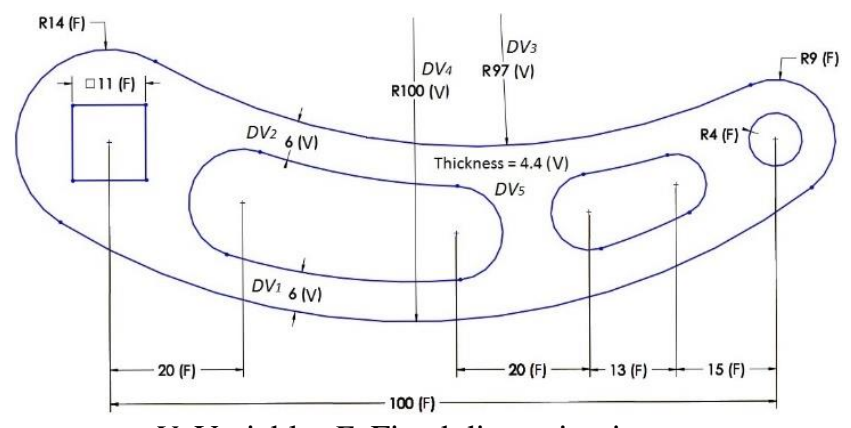

$V$ : Variable. $F$ : Fixed dimension in $\mathrm{mm}$

Fig. 2 The initial geometrical dimensions of the handle

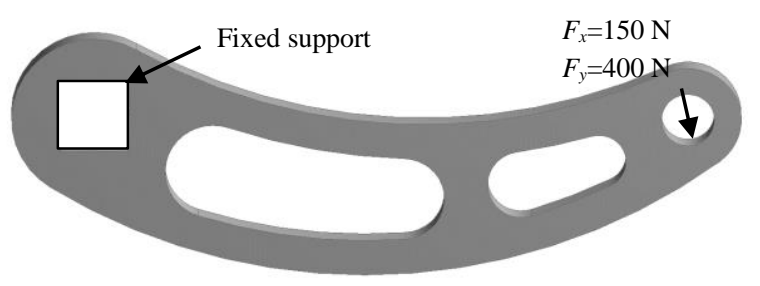

Fig. 3 The boundary conditions of the handle

The lower and upper bounds of the design variables and the initial values of the design are presented in Table 1 . Depending on the bounds of the design variables, a set of 101 samples consisting of the five $D V$ is established using the factorial design technique, and the corresponding two $D R$ (safety factor and mass) are computationally generated using FEM simulations.

Table 1

The lower and upper bounds of the design variables and the initial values of the design

\begin{tabular}{|c|c|c|c|}
\hline & \multicolumn{3}{|c|}{ Design variables } \\
\hline & Symbol & Initial & Limit values \\
\hline Distance & $D V_{1}$ & 6 & $5 \leq D V_{I} \leq 7$ \\
\hline Distance & $D V_{2}$ & 6 & $5 \leq D V_{2} \leq 7$ \\
\hline Radius & $D V_{3}$ & 97 & $85 \leq D V_{3} \leq 120$ \\
\hline Radius & $D V_{4}$ & 100 & $90 \leq D V_{4} \leq 130$ \\
\hline Thickness & $D V_{5}$ & 4.4 & $4 \leq D V_{5} \leq 6$ \\
\hline
\end{tabular}

75 of the 101 samples generated is used to train the ANN model, the rest is used to test the accuracy of the ANN model. At the result of the network training, a promising ANN model, which is built based on the algorithm of feed forward back propagation, has three hidden layers, consisting of 12, 16 and 4 neurons, respectively. A strong correlation with high R2 values of 0.97 and 0.99 is achieved for the testing set of safety factor and mass, respectively (Fig. 4). In other words, the best ANN model confirm that one can generate accurate values of safety factor and mass without FEM simulations by utilizing the ANN model because the testing results of the ANN model is found to be a good agreement with the FEM simulations. Moreover, by means of this model, it is possible to generate a large number of samples of design variables-responses, and to faster search for the best optimum design point among these many samples when compared to that of FEM simulations. 


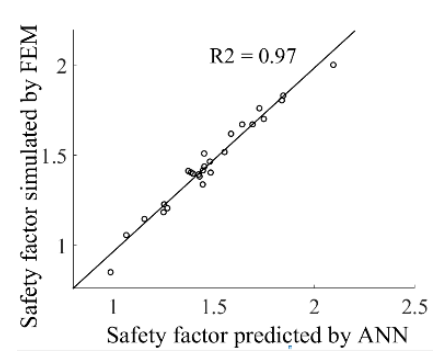

a

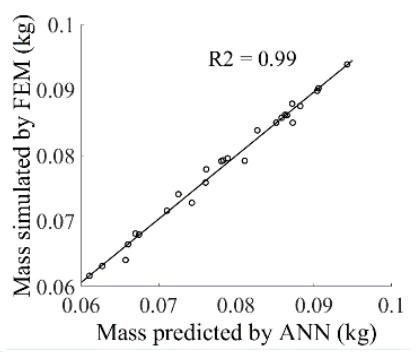

b
Fig. 4 R2 values achieved for the testing set: a) for safety factor, b) for mass

After obtaining the surrogate model based on ANN, the next step is to realize the deterministic design optimization to find nominal or mean values of design variables. To that end, uniform distribution is assigned to each design variable according to their ranges with predetermined lower and upper bounds given in Table 1, and then Monte-Carlo simulations with 10,000 realizations are implemented. The initial and optimal/nominal mean values of design variables found ( $\mu_{D V}^{i n i}$ and $\mu_{D V}^{n}$ ) are presented in Table 2. Compared to the initial design variables, higher safety factor and lower mass values are obtained by the deterministic optimization method. Herein, the starting point of the stochastic design optimization is a critical point. For that end, the deterministically obtained design point $\left(\mu_{D V}^{n}\right)$ can be a reasonable and rational choice as a starting point. Accordingly, the limit-state function can be defined as $P_{v}\left(D R_{1} \leq 1.4982 \vee D R_{2} \geq 0.0662\right) \leq 0$, which refers to nearly zero probabilities that the safety factor is lower than 1.4982 and the mass is higher than $0.0662 \mathrm{~kg}$ under uncertainty conditions. By doing so, it may be possible to find design solutions that are more robust and reliable at worstcase compared to those of the deterministic case.

Optimal design variables and responses found

by deterministic optimization

\begin{tabular}{|c|c|c|c|}
\hline & \multicolumn{3}{|c|}{ Design variables and responses } \\
\hline & Symbol & Initial & $\mu_{D V}^{n}, D R$ \\
\hline Distance & $D V_{1}$ & 6 & 5.4915 \\
\hline Distance & $D V_{2}$ & 6 & 6.9934 \\
\hline Radius & $D V_{3}$ & 97 & 119.7627 \\
\hline Radius & $D V_{4}$ & 100 & 129.9424 \\
\hline Thickness & $D V_{5}$ & 4.4 & 4.1992 \\
\hline Safety factor & $D R_{1}$ & 1.2689 & 1.4982 \\
\hline Mass & $D R_{2}$ & 0.0692 & 0.0662 \\
\hline
\end{tabular}

n Step 4, the stochastic characteristics of $D V$ of the handle are assumed to be Gaussian distribution and a variation subjected to a $\delta_{D V}$ of 0.0001 for each $D V$. The weights of the correlation coefficient for safety factor and mass responses are assumed to be equal importance (0.5 and 0.5). In consideration of these given and assumed values, $\rho_{w_{D V_{i}}}$, $\sigma_{D V i}$ and $S_{D V_{i}}$ are easily calculated via Eq. (5). All of the results of these calculations are presented in Table 3 .
Table 3

The results of search vector and other parameters

\begin{tabular}{|c|c|c|c|c|c|c|c|}
\hline$D V_{i}$ & $\begin{array}{c}0.5 \\
D R_{1} \\
\rho_{11} \\
\end{array}$ & $\begin{array}{c}0.5 \\
D R_{2} \\
\rho_{12} \\
\end{array}$ & $\rho_{w_{D V_{i}}}$ & $\mu_{D V}^{n}$ & $\delta_{D V}$ & $\sigma_{D V i}$ & $S_{D V_{i}}$ \\
\hline$D V_{l}$ & 0.280 & 0.261 & 0.279 & 5.491 & 0.0001 & 0.000549 & 0.000148 \\
\hline$D V_{2}$ & 0.565 & 0.136 & 0.544 & 6.993 & 0.0001 & 0.000699 & 0.000245 \\
\hline$D V_{3}$ & \begin{tabular}{|l|}
0.125 \\
\end{tabular} & -0.133 & 0.112 & 119.762 & 0.0001 & 0.011976 & $-5.1 \mathrm{E}-05$ \\
\hline$D V_{4}$ & -0.079 & 0.059 & -0.071 & 129.942 & 0.0001 & 0.012994 & -0.000126 \\
\hline$D V_{5}$ & 0.543 & 0.943 & 0.562 & 4.199 & 0.0001 & 0.00042 & 0.000311 \\
\hline
\end{tabular}

In the last step, first, the RBRDO formulation in Eq. (6) is rewritten in consideration with the given or found variables, responses and constraints of the design problem, which is given below:

$$
\left.\begin{array}{l}
\operatorname{Min}\left(\mu_{D V_{i}}^{r}, \sigma_{D V_{i}}, \lambda_{s}\right) i=1,2,3,4 \quad \lambda_{s}=1, \ldots, \min _{\lambda_{s}} . \\
\text { Subject to : } \\
P_{v}\left(D R_{1} \leq 1.4982 \vee D R_{2} \geq 0.0662\right) \leq 0 .
\end{array}\right\}
$$

In the optimization definition, the constraint of $P_{v}\left(D R_{1} \leq 1.4982 \vee D R_{2} \geq 0.0662\right) \leq 0$ is specified according to the deterministically found optimal results: the safety factor of 1.4982 and the mass of $0.0662 \mathrm{~kg}$. Here, the primary focus is to investigate the impact of uncertainties in the design variables on the deterministically optimized design responses, and also how to improve reliability and robustness of the deterministically optimum design under uncertainty. For this purpose, to reflect the uncertainty to the five design variables, it is assumed that the mean of each design variable is of a coefficient of variation of 0.0001 stemming from manufacturing or other reasons. Under these conditions, when the described procedure in Step 5 is employed, the optimization results of probability of violation $\left(P_{v}\right)$ and scaling factor $\left(\lambda_{s}\right)$ are obtained (Table 4 and 5). According to Table 4 and 5, an optimum robust design point can be selected among the points where the $P_{v}=0$. Moreover, to decide that, a cost function that is the ratio of mass to safety factor is considered, and the point(s) where the cost function has the lowest value is chosen as the most reliable and robust design point. Accordingly, the optimum point is found to be the intersection between the safety factor of 1.4972 and the mass of $0.0667 \mathrm{~kg}$. The scaling factor $\left(\lambda_{s}\right)$ at that point is found to be 44 . When the formula of $\mu_{D V}^{r}$ in Eq. (6) is employed, the robust mean values of design variables and the corresponding responses are easily calculated.

In addition, to explicitly show the found design solution to be more robust and reliable compared with the initial and deterministic design values, the $P_{v}$ values for $\mu_{D V}^{i n i}$ and $\mu_{D V}^{n}$ at the same constraint as that of the robust solution $\left(P_{v}\left(D R_{1} \leq 1.4982 \vee D R_{2} \geq 0.0662\right)\right.$ are separately computed using Monte-Carlo simulations with 1000 realizations. All of these calculation results are presented in Table 6 . 
Table 4

The results of the probability of violation $\left(P_{v}\right)$ calculation

\begin{tabular}{|c|c|c|c|c|c|c|c|c|c|c|}
\hline \multirow{2}{*}{\multicolumn{2}{|c|}{$P_{v}$}} & \multicolumn{9}{|c|}{ Mass } \\
\hline & & 0.0662 & 0.0663 & 0.0664 & 0.0665 & 0.0666 & 0.0667 & 0.0668 & 0.0669 & 0.067 \\
\hline \multirow{9}{*}{ 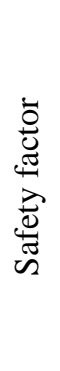 } & 1.4982 & 0.921 & 0.547 & 0.204 & 0.0580 & 0.0090 & 0.0010 & 0 & 0 & 0 \\
\hline & 1.4972 & 0.839 & 0.417 & 0.153 & 0.0420 & 0.0040 & 0 & 0 & 0 & 0 \\
\hline & 1.4962 & 0.746 & 0.341 & 0.109 & 0.0170 & 0.0020 & 0 & 0 & 0 & 0 \\
\hline & 1.4952 & 0.687 & 0.251 & 0.0580 & 0.0110 & 0.0020 & 0 & 0 & 0 & 0 \\
\hline & 1.4942 & 0.627 & 0.203 & 0.0490 & 0.0050 & 0 & 0 & 0 & 0 & 0 \\
\hline & 1.4932 & 0.596 & 0.154 & 0.0300 & 0.0040 & 0 & 0 & 0 & 0 & 0 \\
\hline & 1.4922 & 0.585 & 0.134 & 0.0160 & 0 & 0 & 0 & 0 & 0 & 0 \\
\hline & 1.4912 & 0.575 & 0.145 & 0.0140 & 0.0010 & 0 & 0 & 0 & 0 & 0 \\
\hline & 1.4902 & 0.539 & 0.126 & 0.0090 & 0 & 0 & 0 & 0 & 0 & 0 \\
\hline
\end{tabular}

Table 5

The optimization results of the scaling factor $\left(\lambda_{s}\right)$ calculation

\begin{tabular}{|c|c|c|c|c|c|c|c|c|c|c|}
\hline \multirow{2}{*}{\multicolumn{2}{|c|}{$\lambda_{s}$}} & \multicolumn{9}{|c|}{ Mass } \\
\hline & & 0.0662 & 0.0663 & 0.0664 & 0.0665 & 0.0666 & 0.0667 & 0.0668 & 0.0669 & 0.067 \\
\hline \multirow{9}{*}{ 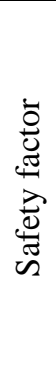 } & 1.4982 & 3 & 9 & 19 & 25 & 36 & 38 & 44 & 44 & 48 \\
\hline & 1.4972 & 1 & 6 & 13 & 28 & 34 & 44 & 45 & 36 & 37 \\
\hline & 1.4962 & 1 & 6 & 16 & 21 & 35 & 33 & 37 & 35 & 36 \\
\hline & 1.4952 & 1 & 4 & 8 & 19 & 27 & 26 & 29 & 24 & 31 \\
\hline & 1.4942 & 1 & 1 & 9 & 17 & 18 & 24 & 24 & 24 & 25 \\
\hline & 1.4932 & 1 & 1 & 4 & 12 & 18 & 17 & 17 & 21 & 20 \\
\hline & 1.4922 & 1 & 1 & 2 & 11 & 9 & 9 & 9 & 11 & 10 \\
\hline & 1.4912 & 1 & 1 & 1 & 4 & 8 & 6 & 6 & 2 & 9 \\
\hline & 1.4902 & 1 & 1 & 1 & 4 & 3 & 2 & 1 & 2 & 3 \\
\hline
\end{tabular}

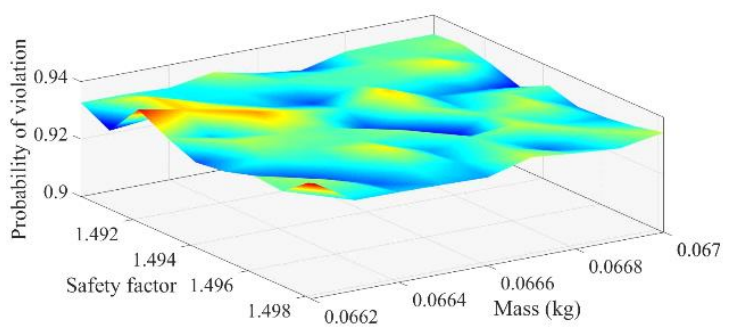

a

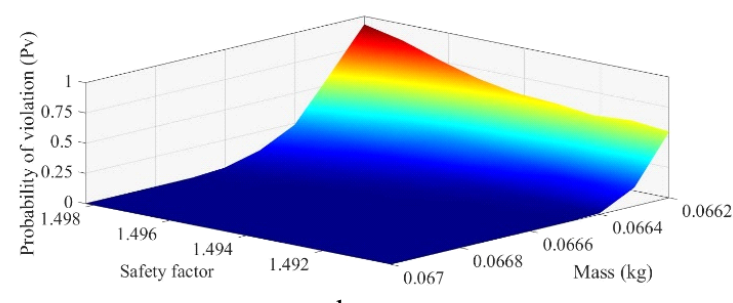

b
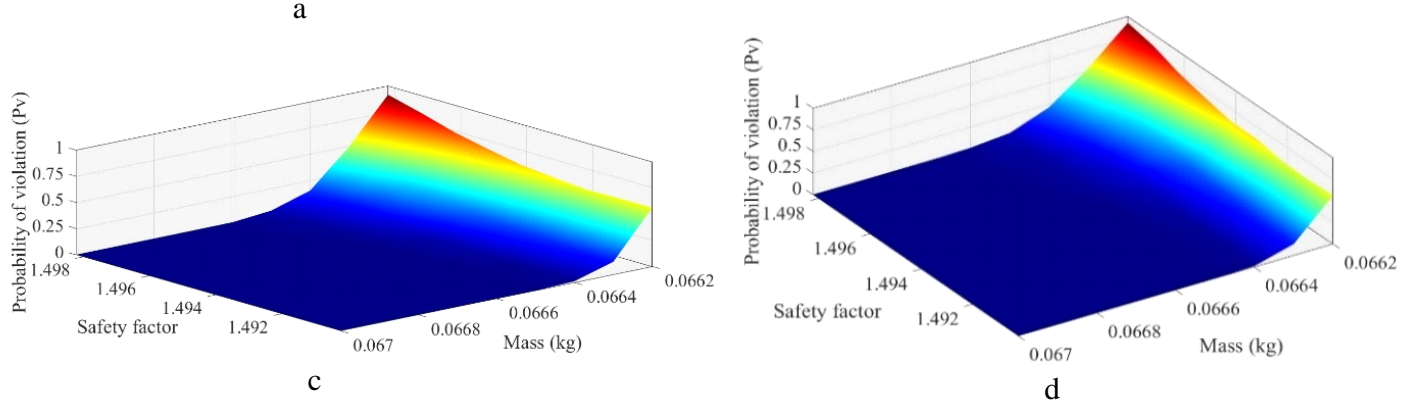

Fig. 5 The 3D graph representation of different optimization cases with different relative importances: a) The case without implementing optimization, b) $5 \%$ SF-95\% M, c) $95 \%$ SF-5\% M, d) $50 \%$ SF-50\% M

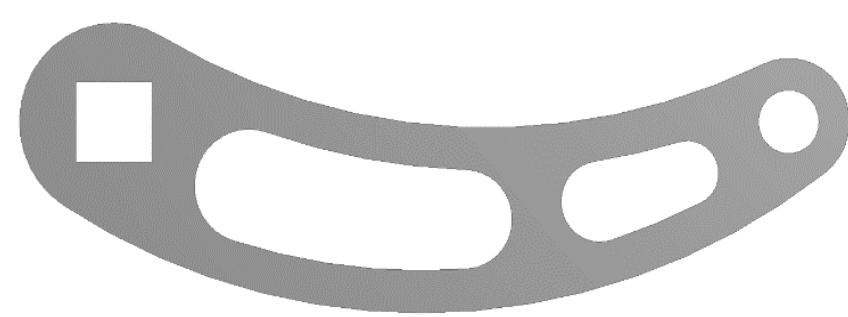

a

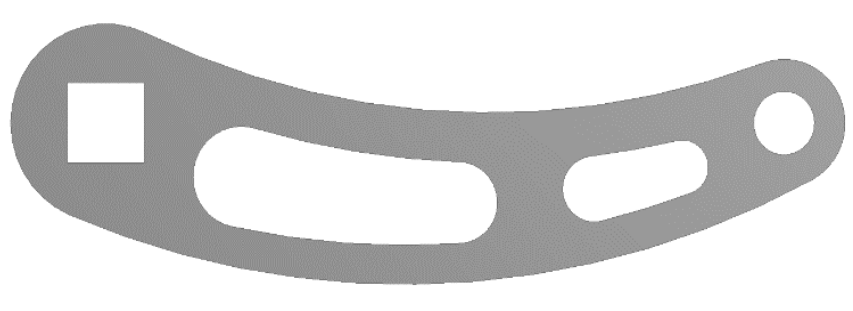

b

Fig. 6 The solid geometries of initial and robust design solutions: a) initial design, b) robust design 
As seen in the Table, the robust solution ensures a $P_{v}$ of zero, whereas the initial and deterministic optimal solution can only ensure a $P_{v}$ of 1.0 and 0.41 , respectively at the same constraint conditions. Also, this robust design searching process takes only about 20 seconds. Therefore, all of the results demonstrates that the proposed method enables designers to efficiently and effectively find reliable and robust designs under uncertainty.

\section{Table 6}

The comparison of the initial, nominal and robust design solutions

\begin{tabular}{|c|c|c|c|}
\hline & $\mu_{D V}^{i n i}$ & $\mu_{D V}^{n}$ & $\mu_{D V}^{r}, D R$ \\
\hline$D V_{1}$ & 6 & 5.491 & 5.4931 \\
\hline$D V_{2}$ & 6 & 6.993 & 6.9961 \\
\hline$D V_{3}$ & 97 & 119.762 & 119.762 \\
\hline$D V_{4}$ & 100 & 129.942 & 129.941 \\
\hline$D V_{\underline{5}}$ & 4.4 & 4.199 & 4.2026 \\
\hline$D R_{1}$ & 0.0692 & 0.0662 & 0.0662 \\
\hline$D R_{2}$ & 1.2689 & 1.4982 & 1.5061 \\
\hline$\lambda_{s}$ & - & - & 44 \\
\hline$P_{v}$ & 1.0 & 0.41 & 0 \\
\hline
\end{tabular}

The proposed method also provides designers with a flexibility to decide against the relative importance of the design response criteria. In other words, in the design example, it is possible to give more importance to safety factor or mass before implementing the robust optimization. In Fig. 5 , the case without implementing optimization, the case of $5 \% \mathrm{SF}-95 \% \mathrm{M}$, the case of $95 \% \mathrm{SF}-5 \% \mathrm{M}$, and the case of $50 \%$ SF- $50 \%$ M (SF: safety factor, M: mass) is illustrated by the $3 \mathrm{D}$ graph. The found robust optimization solution above is considered in the case of $50 \% \mathrm{SF}-50 \% \mathrm{M}$, and the solid geometries of initial and robust design solutions are shown in Fig. 6.

\section{Conclusion}

In this work, an efficient simulation-based search method for reliability-based robust design optimization is proposed, which consisting of five steps: 1. Generating a sample set for design variables and responses from FEM; 2. Building a surrogate model representing the sample set by using ANN; 3. Optimizing the design deterministically via MCS; 4. Determining stochastic characteristics and calculating the search vector; 5. Optimizing the design under uncertainty via MCS. This proposed method is applied to the design optimization of a car handle to show its effectiveness and efficacy. The searching or optimization process took only 20 seconds. Results demonstrated that the proposed method enable designers to efficiently and effectively find reliable and robust designs under uncertainty, and also provides designers with a flexibility to decide about the relative importance of the design response criteria. To enhance this method, some new directions on this area can be suggested: 1. Integrating this method with heuristic optimization methods such as genetic algorithm and particle swarm optimization algorithm; 2 . Applying this method in the complex system designs.

\section{References}

1. Yang, H.; Zhu, Y.; Lu, Q.; Zhang, J. 2015. Dynamic reliability based design optimization of the tripod substructure of offshore wind turbines, Renewable Energy 78: 16-25. http://dx.doi.org/10.1016/j.renene.2014.12.061.

2. Allen, M.; Maute, K. 2004. Reliability-based design optimization of aeroelastic structures, Structural and Multidisciplinary Optimization 27(4): 228-242. http://dx.doi.org/10.1007/s00158-004-0384-1.

3. Xia, B.; Lü, H.; Yu, D., and Jiang, C. 2015. Reliability-based design optimization of structural systems under hybrid probabilistic and interval model, Computers \& Structures 160: 126-134.

http://dx.doi.org/10.1016/j.compstruc.2015.08.009.

4. Dubourg, V.; Sudret, B., and Bourinet, J.-M. 2011. Reliability-based design optimization using kriging surrogates and subset simulation, Structural and Multidisciplinary Optimization 44(5): 673-690. http://dx.doi.org/10.1007/s00158-011-0653-8.

5. Zang, C.; Friswell, M.I.; Mottershead, J.E. 2005. A review of robust optimal design and its application in dynamics, Computers \& Structures 83(4-5): 315-326. http://dx.doi.org/10.1016/j.compstruc.2004.10.007.

6. Shah, H.; Hosder, S.; Koziel, S.; Tesfahunegn, Y.A., and Leifsson, L. 2015. Multi-fidelity robust aerodynamic design optimization under mixed uncertainty, Aerospace Science and Technology 45: 1729. http://dx.doi.org/10.1016/j.ast.2015.04.011.

7. Wu, J.; Gao, J.; Luo, Z.; Brown, T. 2016. Robust topology optimization for structures under interval uncertainty, Advances in Engineering Software 99: 3648.

http://dx.doi.org/10.1016/j.advengsoft.2016.05.002.

8. Harry, M.J.1997.The nature of Six Sigma Quality. Shaumburg: IL: Motorola University Press.

9. Taguchi, G.; Chowdhury, S.; Taguchi, S.2000.Robust Engineering. New York: McGraw Hill.

10. Taguchi, G. 1993.Taguchi on robust technology development: Bringing qualityengineering upstream. New York: New York: ASME Press. https://doi.org/10.1115/1.800288.

11. Lima, R.; Soize, C.; Sampaio, R. 2015. Robust design optimization with an uncertain model of a nonlinear vibro-impact electro-mechanical system, Communications in Nonlinear Science and Numerical Simulation 23 (1-3): 263-273. http://dx.doi.org/10.1016/j.cnsns.2014.11.014.

12. Zaman, K.; McDonald, M.; Mahadevan, S.; Green, L. 2011. Robustness-based design optimization under data uncertainty, Structural and Multidisciplinary Optimization 44(2): 183-197. http://dx.doi.org/10.1007/s00158-011-0622-2.

13. Tammareddi, S.; Sun, G.; Li, Q. 2016. Multiobjective robust optimization of coronary stents, Materials \& Design 90: 682-692. http://dx.doi.org/10.1016/j.matdes.2015.10.153.

14. Stocki, R.; Szolc, T.; Tauzowski, P.; Knabel, J. 2012. Robust design optimization of the vibrating rotor-shaft system subjected to selected dynamic constraints, Mechanical Systems and Signal Processing 29: 34-44. http://dx.doi.org/10.1016/j.ymssp.2011.07.023. 
15. Forouzandeh Shahraki, A.; Noorossana, R. 2014. Reliability-based robust design optimization: A general methodology using genetic algorithm, Computers \& Industrial Engineering 74: 199-207. http://dx.doi.org/10.1016/j.cie.2014.05.013.

16. Wang, P.; Wang, Z.; Youn, B.D.; Lee, S. 2015. Reliability-based robust design of smart sensing systems for failure diagnostics using piezoelectric materials, Computers \& Structures 156: 110-121. http://dx.doi.org/10.1016/j.compstruc.2015.04.012.

17. Gang, W.; Wang, S.; Augenbroe, G.; Xiao, F. 2016. Robust optimal design of district cooling systems and the impacts of uncertainty and reliability, Energy and Buildings 122: 11-22. http://dx.doi.org/10.1016/j.enbuild.2016.04.012.

18. Choi, S.-K.; Grandhi, R.V.; Canfield, R.A.2007.Reliability-based Structural Design. Springer-Verlag London.

19. Li, F.; Sun, G.; Huang, X.; Rong, J.; Li, Q. 2015. Multiobjective robust optimization for crashworthiness design of foam filled thin-walled structures with random and interval uncertainties, Engineering Structures 88: 111-124. http://dx.doi.org/10.1016/j.engstruct.2015.01.023.

20. Díaz, J.; Cid Montoya, M., and Hernández, S. 2016. Efficient methodologies for reliability-based design optimization of composite panels, Advances in Engineering Software 93: 9-21. http://dx.doi.org/10.1016/j.advengsoft.2015.12.001.

21. Seshadri, P.; Constantine, P.; Iaccarino, G.; Parks, G. 2016. A density-matching approach for optimization under uncertainty, Computer Methods in Applied Mechanics and Engineering 305: 562-578. http://dx.doi.org/10.1016/j.cma.2016.03.006.

22. Fang, J.; Gao, Y.; Sun, G.; Xu, C.; Li, Q. 2015. Multiobjective robust design optimization of fatigue life for a truck cab, Reliability Engineering \& System Safety 135: $1-8$.

http://dx.doi.org/10.1016/j.ress.2014.10.007.
23. Paiva, R.M.; Crawford, C.; Suleman, A. 2014. Robust and Reliability-Based Design Optimization Framework for Wing Design, AIAA Journal 52: 711-724. http://dx.doi.org/10.2514/1.J052161.

\section{Mayda}

\section{AN EFFICIENT SIMULATION-BASED SEARCH METHOD FOR RELIABILITY-BASED ROBUST DESIGN OPTIMIZATION OF MECHANICAL COMPONENTS}

S u m m a r y

Reliability-based robust design optimization (RBRDO) aims to minimize the variation in the system, and ensure the levels of failure probability of the system. Despite significant improvements on RBRDO, several challenges have been emerging. First, the existing implementations of RBRDO are complex to apply them to design problems. Second, an efficient method of optimum search is needed to enhance the RBRDO process. To address these issues, in this work, a simulation-based search method for RBRDO is proposed by utilizing Monte-Carlo Simulation and Artificial Neural Network. Specifically, to accurately select an optimum searching direction and step lengths, a search vector based on correlation coefficients between design variables and responses is put forward. This proposed method is applied to the design of a car handle to show its effectiveness and efficacy. Results demonstrates that this method enables to efficiently and effectively find reliable and robust designs under uncertainty compared to the deterministic case.

Keywords: Monte Carlo method, reliability, robust design optimization, search method.

Received July 07, 2016

Accepted October 13, 2017 